Les livres

Saint Pol (Thibaud de). - Le corps désirable. Hommes et femmes face à leur poids.

Paris, Presses Universitaires de France (Le lien social), 2010, 224 p., $25 €$.

L'ouvrage de Thibault de Saint Pol est le bienvenu pour au moins trois raisons. 
D'abord parce qu'il était temps que s'affirme un autre regard sur ce qui s'est imposé en problème de santé public majeur - l'obésité -, tant les cadrages biomédicaux ou sanitaires dominent, pour les saturer, l'espace public et le marché des savoirs scientifiques sur le sujet. On en aurait presque oublié ce que Mauss répétait à l'envie : le corps n'est pas qu'un système complexe de tissus, d'organes ou de chair, mais bien le « miroir d'une époque et de son fonctionnement» (p. 2). Ensuite, parce que ce problème social est l'objet idéal permettant d'illustrer la fécondité d'une perspective qui accorde au genre l'importance essentielle qu'il mérite: le genre s'affirme en effet, dans l'ouvrage, comme un aspect-clé de compréhension du rapport que l'on entretient au corps et à la corpulence. C'est également par le prisme du genre qu'un thème qui croît chaque jour davantage en audience politique, celui des inégalités sociales de santé, est minutieusement exploré : saisir les dynamiques au principe des inégalités de corpulence aide à mieux « comprendre les inégalités dans leur globalité » (p. 5). Enfin, parce qu'il importe, selon l'auteur, que la sociologie se (re)donne le corps comme objet principal, et qu'elle cesse d'ignorer qu'il est un essentiel « composant de l'action humaine » (p. 2) : si l'on accepte que les relations que nous nouons avec les autres et notre environnement sont médiatisées par notre corps, il devient impossible «de construire une théorie de l'action sans prendre en compte le corps» (p. 3).

Pour mener à bien son analyse, Saint Pol exploite, de manière souvent inédite et parfois originale, un vaste corpus de données quantitatives, lesquelles permettent d'atteindre un niveau satisfaisant et nécessaire de représentativité et de recul. Il n'existe guère, selon l'auteur, d'enquêtes exhaustives, ce qui le conduit à utiliser et combiner, selon les exigences de l'analyse, différentes sources complémentaires. L'ouvrage s'appuie ainsi sur les résultats issus de l'exploitation de huit grandes enquêtes quantitatives, au premier rang desquelles l'on trouve le «Panel européen des ménages » que réalise chaque année l'Union européenne. Pour ce qui est du cas spécifique de la France, Saint Pol se fonde sur les résultats de nombreuses études récentes, et en particulier des enquêtes "Santé » réalisées par l'Insee. Finalement, afin de "pouvoir se pencher plus précisément sur les rapports des individus à leur corps » (p.10), il utilise l'enquête « Histoire de vie » réalisée par l'Insee en 2003. L'auteur se donne, en somme, les moyens empiriques de ses ambitions: une recherche conçue comme pluridisciplinaire, en ce qu'elle entend dialoguer avec des disciplines aussi diverses que la biologie, la médecine ou la nutrition, couvrant une vaste période historique (vingt-cinq ans), et comparative, puisqu' elle confronte la situation française à celles des autres pays européens.

L'ouvrage s'épanouit dans la succession de quatre chapitres principaux, lesquels scandent les étapes d'un raisonnement élégant et logique. Dans une première partie, Saint Pol revient sur les processus de construction sociale des corps. Il s'attache en particulier, dans une veine sociologique désormais classique, à montrer comment les différences sociales qui génèrent des habitudes et des modes de vie différents s'impriment dans les corps, au point que le corps ainsi marqué soit aussi un "marqueur d'appartenance ». L'auteur insiste sur le fait que l'expérience que les différentes classes sociales ont de leur corps est variable et que celle-ci induit un rapport à l'alimentation également fort différent, les classes populaires, par exemple, valorisant les aliments nourrissants et fortifiants, comme l'avait déjà remarqué Boltanski dans un article pionnier. Mais ce qu'il y a aujourd'hui de nouveau, c'est la montée des exigences sanitaires et du souci de santé : le corps est devenu un capital à faire fructifier, une ressource à choyer, un patrimoine (presque) comme les autres (Le Breton), désormais objet 
autant que cible de nombreuses interventions individuelles et publiques.

Dans une deuxième partie, Saint Pol, dans la lignée des travaux néo-foucaldiens (voir Peter Miller et Nikolas Rose), montre comment, avant que l'obésité devienne une catégorie d'action publique, ont été élaborés des instruments de mesure, et se sont épanouies des statistiques et leurs moyennes, dessinant les formes de la normalité et de l'anormalité des corps. Analysant les différentes solutions historiques de mesure de la taille puis du poids, brossant ainsi un vaste panorama allant des travaux originels de Buffon et de Quételet jusqu'aux unités de mesures contemporaines, il indique comment le poids s'affirme progressivement comme une variable sur laquelle, contrairement à la taille, on peut agir et sur laquelle il faut agir. Après avoir ainsi passé en revue de nombreuses formules de calcul du poids idéal, Saint Pol accorde une place de choix à la genèse et à l'étude de l'Indice de masse corporelle (IMC) et aux raisons qui ont conduit les scientifiques et les organisations internationales à l'imposer comme catégorie de mesure universelle. S'il existe peu d'attributs physiques disposant «d'un instrument qui permet leur mesure et leur analyse sur une large population » (p. 48), Saint Pol avertit cependant qu'il ne compose pas une mesure neutre, bien qu'il passe souvent pour «scientifique ", et que cet indice, certes mondialement accepté, n'en « conserve pas moins une marge d'erreur» (p. 75).

C'est dans la troisième partie que Saint Pol exploite de manière plus systématique les données quantitatives qu'il a réunies. Partant de l'hypothèse que les perceptions du corps qu'a un individu particulier sont «influencées par les représentations et les normes de la société à laquelle il appartient» (p. 89), l'auteur commence par revenir sur les représentations successives qu'a connues, au cours des âges, le « corps idéal » et sur quelques-unes des dynamiques historiques qui ont présidé à l'évolution des standards esthétiques. Il compare, à cette occasion, les normes qui ont exercé leur «tyrannie » sur les hommes et celles qui se sont imposées aux femmes, remarquant que la «contrainte pondérale» pèse plus intensément sur les secondes que sur les premiers. Il conclut que, contrairement à certaines analyses rapides, on ne serait pas récemment passé, en Occident, d'une société valorisant l'obésité à une autre qui célébrerait la minceur, mais que c'est plutôt «le seuil de ce qui apparaît comme obésité qui a diminué »(p. 96). Sur ces bases historiques, il brosse un panorama détaillé de la situation de la corpulence dans le monde, en Europe, et en France. Il montre que «l'éventail des valeurs prises par la corpulence est assez similaire pour les hommes d'un pays à l'autre, mais nettement plus varié pour les femmes » (p. 105). Il souligne également que si la France possède une corpulence parmi les plus faibles des pays développés, il existe cependant dans ce pays un écart relativement important entre les hommes et les femmes. Il pointe ainsi un constat d'importance : "L'obésité est une pathologie plutôt féminine que masculine, du moins dans ses cas les plus sévères. » (p. 109), tandis que l'embonpoint serait, lui, un fait social plutôt masculin. Il ne se contente pas, toutefois, d'objectiver et de comparer des états corporels physiques; il double cette analyse d'une étude des représentations que se font les individus de leur poids (poids perçu et désiré). Fidèle à ces ambitions initiales, il insiste volontiers sur les différences existant entre les hommes et les femmes à ce sujet, ces dernières témoignant d'une plus grande insatisfaction à l'égard de leur poids. Il ajoute que les normes de poids idéal sont très différentes d'un pays à l'autre: les Français auraient ainsi une corpulence idéale plus faible que dans les autres pays européens, «signe d'une pression plus forte exercée sur la corpulence dans ce pays » (p. 117). Mais que l'on ne s'y trompe pas: en dépit des variations locales, contrôler son 
poids est devenu, partout en Europe, un nouvel « usage obligé du corps ». Et d'en conclure que «la libération des corps se manifeste paradoxalement par une forte contrainte, des efforts et une perpétuelle surveillance de soi et de son alimentation » (p. 121).

La quatrième partie s'attaque à ce qui constitue certainement le cœur des intérêts scientifiques de l'auteur : étudier les inégalités sociales de corpulence, et évaluer, en particulier, le poids du genre dans la genèse de pareilles inégalités. À ces fins, l'auteur analyse quelles caractéristiques individuelles (diplôme, revenu, statut marital, etc.) jouent un rôle décisif, quoique variable, dans la formation des inégalités sociales face aux poids et insiste sur le fait que ces caractéristiques ne jouent pas de manière comparable selon que l'on est un homme ou une femme. S'il n'est guère possible d'énumérer l'ensemble des constats fort intéressants dressés ici, on peut toutefois relever que plus on est diplômé, plus la corpulence est faible, et que l'écart « entre les moins diplômés et plus diplômés est beaucoup plus élevé pour les femmes que pour les hommes » (p. 132). D’une manière générale, Saint Pol identifie un «lien entre une corpulence des femmes élevée et un faible statut socioéconomique » (p. 144) : s'il est vrai que l'on trouve une surreprésentation des obèses chez les ménages les plus pauvres et que l'écart avec les plus aisés a eu tendance à (légèrement) augmenter entre 1990 et le milieu des années 2000, le lien entre obésité et appartenance aux milieux modestes est surtout valable, en France, pour les femmes. Comment expliquer cette distribution sélective de la corpulence dans les différents milieux sociaux ? Saint Pol estime que le faible coût des aliments les plus caloriques « est sans doute pour beaucoup dans la plus forte prévalence de l'obésité chez les populations défavorisées » (p. 152). Plus généralement, la brusque augmentation de la prévalence de l'obésité en France tiendrait au changement de la structure des apports personnels, et en particulier au développement de la consommation des plats cuisinés et à la multiplication des pratiques de grignotage. Après avoir révélé la puissance des déterminants socioéconomiques et de genre dans la genèse des inégalités sociales face au poids, Saint Pol entreprend une critique, salutaire mais convenue, des discours médicaux, et de leur monopole comme fondements des discours médiatiques. Il insiste, en outre, sur les discriminations et stigmatisations dont font l'objet les personnes en surpoids et obèses, l'obésité fonctionnant comme un stigmate surtout, mais pas seulement, sur le marché du travail : ce qu'il nomme les «inégalités corporelles » redouble ainsi les inégalités économiques et culturelles. L'auteur attire l'attention sur le fait que les discriminations «sont moins graves pour les hommes » qu'elles ne le sont pour les femmes, la « situation des hommes en surpoids n'étant pas significativement différente des hommes de poids "normaux" " (p. 173). Et Saint Pol de conclure : «Loin de ne présenter qu'un enjeu de santé publique, les disparités de corpulence traduisent ainsi des inégalités, entre groupes sociaux, mais aussi entre sexes.»(p. 191). Ces arguments lui permettent de terminer sur la nécessité, quand on dessine des instruments d'action publique, de prendre acte des déterminants socioéconomiques, culturels et de genre, une politique publique «efficace » devant prendre en considération la façon dont chacun des deux genres se représente, différemment, le corps désirable.

Le livre est clair, bien écrit, sans verbiage et atteint, parfois avec brio, la plupart de ses objectifs principaux. Toutefois, bien que Saint Pol prenne soin de discuter l'opportunité de l'usage du terme d'épidémie et qu'il critique l'omniprésence du discours médical dans l'espace public, on ne peut manquer de regretter que l'obésité comme problème 
social soit, dans l'ensemble, tenu pour acquis, et qu'il ne soit pas fait mention des nombreux débats et controverses qui portent, notamment aux États-Unis (voir Abigail Saguy), sur l'importance « réelle » du phénomène et de ses conséquences. Dans la même veine, si on ne peut qu'admirer la maîtrise des savoirs techniques qu'affiche l'auteur dans les pages traitant des différentes mesures de la corpulence, on regrette qu'il se fonde principalement sur des arguments technicistes (de statistiques et de praticité) pour justifier de la préférence finalement accordée à l'IMC sur les mesures alternatives : on aurait aimé une approche un peu plus critique et constructiviste du choix de la mesure et que les rôles de l'OMS et de l'IOTF, par exemple, ne fussent pas présentés « à plat », c'est-àdire comme des prescripteurs neutres, là où d'autres travaux ont une approche plus politique de la nature de leur activité. Finalement, si l'ouvrage réussit à dénaturaliser l'objet «obésité », en montrant ce que son développement doit «à la société », il est moins convaincant dans son analyse politique et nombreuses sont les conclusions auxquelles il parvient qui déjà ont été énoncées, souvent avec plus de force, dans d'autres travaux spécialistes du sujet (voir les écrits de Poulain). On aurait aimé, enfin, que l'ouvrage honorât plus parfaitement l'une de ses ambitions affichées dans l'introduction: Thibaud de Saint Pol affirme certes que le corps est un composant essentiel de l'action humaine, mais on peine, en réalité, à saisir précisément, c'est-à-dire de manière explicitement élaborée, quelles sont les conséquences de cette affirmation pour la théorie sociologique, et en particulier pour la théorie de l'action.

\section{Henri Bergeron}

Centre de sociologie des organisations Cnrs-Sciences Po 